\title{
The Role of 39 Psoriasis Risk Variants on Age of Psoriasis Onset
}

\author{
Yingchang Lu, ${ }^{1}$ Sinae Kane, ${ }^{1}$ Haoyan Chen, ${ }^{1,2}$ Argentina Leon, ${ }^{1}$ Ethan Levin, ${ }^{1}$ \\ Tien Nguyen, ${ }^{1}$ Maya Debbaneh, ${ }^{1,3}$ Jillian W. Millsop, ${ }^{1,4}$ Rishu Gupta, ${ }^{1,5}$ Monica Huynh, ${ }^{1,6}$ \\ Daniel Butler, ${ }^{1}$ Kelly Cordoro, ${ }^{1}$ and Wilson Liao ${ }^{1}$
}

${ }^{1}$ Department of Dermatology, University of California San Francisco, 2340 Sutter Street, P.O. Box 0808, San Francisco, CA 94143-0808, USA

${ }^{2}$ Department of Gastroenterology, Ren Ji Hospital, Shanghai Jiao Tong University School of Medicine, Shanghai Institute of Digestive Diseases, Shanghai 200001, China

${ }^{3}$ University of California Irvine School of Medicine, Irvine, CA 92697, USA

${ }^{4}$ University of Utah School of Medicine, Salt Lake City, UT 84132, USA

${ }^{5}$ Keck School of Medicine, University of Southern California, Los Angeles, CA 90089, USA

${ }^{6}$ Chicago College of Osteopathic Medicine, Downers Grove, IL 60515, USA

Correspondence should be addressed to Wilson Liao; liaowi@derm.ucsf.edu

Received 16 July 2013; Accepted 29 August 2013

Academic Editors: B. Amichai, G. Chodorowska, C. Ferrándiz, and A. Tosca

Copyright (C) 2013 Yingchang Lu et al. This is an open access article distributed under the Creative Commons Attribution License, which permits unrestricted use, distribution, and reproduction in any medium, provided the original work is properly cited.

\begin{abstract}
Recent genome-wide association studies (GWAS) have identified multiple genetic risk factors for psoriasis, but data on their association with age of onset have been marginally explored. The goal of this study was to evaluate known risk alleles of psoriasis for association with age of psoriasis onset in three well-defined case-only cohorts totaling 1,498 psoriasis patients. We selected 39 genetic variants from psoriasis GWAS and tested these variants for association with age of psoriasis onset in a meta-analysis. We found that rs10484554 and rs12191877 near HLA-C and rs17716942 near IFIH1 were associated with age of psoriasis onset with false discovery rate $<0.05$. The association between rs17716942 and age of onset was not replicated in a fourth independent cohort of 489 patients $(P=0.94)$. The imputed $H L A-C * 06: 02$ allele demonstrated a much stronger association with age of psoriasis onset than rs10484554 and rs12191877. We conclude that despite the discovery of numerous psoriasis risk alleles, HLA-C*06:02 still plays the most important role in determining the age of onset of psoriasis. Larger studies are needed to evaluate the contribution of other risk alleles, including IFIH1, to age of psoriasis onset.
\end{abstract}

\section{Introduction}

Psoriasis is an inflammatory, immune-mediated disorder of the skin, joints, and nails with an estimated prevalence of $2-3 \%$ of the population. Henseler and Christophers divided psoriasis into two subtypes. Type I psoriasis manifests before age 40 with peak onset at 16-22 years, and Type II psoriasis begins after age 40 with peak onset at 57-60 years [1]. Type I and II psoriasis have been shown to differ clinically in their severity, relapse frequency, and family history $[1,2]$. The clinical differences in Type I and Type II psoriasis are paralleled by genetic differences. Type I psoriasis has a stronger genetic basis as a greater proportion of patients had a family history of psoriasis, and has stronger HLA-C*06 associations; however, Type II psoriasis is negative in family history, and is not associated with $H L A-C * 06[1,3,4]$. Over the past few years, over 36 novel psoriasis loci have been identified through genome-wide association studies (GWAS) [5-13]. However, it is not known to what degree these susceptibility alleles influence the age of onset of psoriasis. Here, we examined 39 of these genetic variants in 3 cohorts of psoriasis cases to ascertain whether any of these loci were preferentially associated with the age of psoriasis onset.

\section{Materials and Methods}

The SNPs were selected from psoriasis GWAS conducted in Caucasian populations (published before December 2012; 
see Supplementary Table 1 available online at http://dx.doi .org/10.1155/2013/203941), taking into account the potential molecular mechanisms underlying the pathogenesis of psoriasis [14]. We evaluated 39 SNPs for association with age of psoriasis onset in two psoriasis GWAS case cohorts; 13 of those SNPs were genotyped by Illumina GoldenGate assay in a third cohort of 418 cases at the University of California San Francisco (UCSF) (Table 1). rs17716942 in IFIH1 was genotyped in a fourth independent cohort of 489 National Psoriasis Foundation (NPF) cases using the Applied Biosystems Taqman genotyping assay. Age of onset of psoriasis was determined by patient self-report and by the treating dermatologist. The two GWAS cohorts consisted of the Genetic Association Information Network (GAIN) cohort including 898 cases [7] and the Washington University/University of California San Francisco cohort (WashU/UCSF) including 182 cases [6]. IMPUTE2 [15] was used to impute the ungenotyped SNPs in these two case cohorts by using phase 3 HapMap and 1000 Genomes pilot project CEU haplotypes as a reference. Since the distributions of the age onset of psoriasis in our four independent case cohorts were not bimodal but positively skewed (Supplementary Figures 1-4), a square-root transformed age onset was used in the analysis. The linear regression quantitative trait test in SNPTEST was used to associate the imputed dosage for each SNP with the age onset of psoriasis separately in GAIN and WashU/UCSF cohorts with the adjustment of gender. Only imputed SNPs with relatively high confidence (PROPER_Info $>0.5$ ) were analyzed [16]. The association results from the GAIN cohort, WashU/UCSF cohort, and UCSF cohort were then combined by meta-analysis using inverse-variance method based on a fixed-effect model. The false discovery rate method was used to correct for multiple testing (FDR_q < 0.05).

\section{Results}

We used linear regression to evaluate the association of 39 psoriasis genetic loci with age of psoriasis onset in a combined sample of 1,498 cases. These loci were identified by previous psoriasis genome-wide association studies and include variants within or near the HLA locus, IL23R, IL12B, TNIP1, TNFAIP3, IL23A, IL13, TRAF3IP2, LCE3B/3C, RNF114, IFIH1, ERAP1, REL, TYK2, NFKBIA, NOS2, IL28RA, SDC4, FBXL19, and RPS26. We found that rs10484554 and rs12191877 near HLA-C and rs17716942 near IFIH1 were associated with age onset of psoriasis with FDR_q value $<0.05$. As expected, the alleles associated with increased risk of psoriasis were also associated with younger age of onset of psoriasis (Table 1). However, the association between rs17716942 and age of psoriasis onset was not replicated in the NPF cases (beta $=-0.01$ and $P=0.94$ for the $\mathrm{T}$ allele, Table 1$)$. Since the top two SNPs associated with age of onset were located near the HLA-C locus, we further imputed HLA$C * 06: 02$ in GAIN, WashU/UCSF, and UCSF case cohorts as previously described [17]. HLA-C*06:02 was more strongly associated with age of onset than the two individual HLA$C$ SNPs (Table 1). Our linear regression model revealed that psoriasis patients with two copies of $H L A-C * 06: 02$ developed psoriasis on average at age 15 , psoriasis patients with one copy of $H L A-C * 06: 02$ developed psoriasis at age 21, and psoriasis patients with zero copies of $H L A-C * 06: 02$ developed psoriasis at age 27 . Thus, each additional copy of $H L A-C * 06: 02$ reduced the onset of psoriasis by 6 years.

\section{Discussion}

In this study we found that among the many psoriasis loci discovered by GWAS, only the $H L A-C$ locus showed robust evidence of playing a role in earlier psoriasis onset. The HLA$C * 06: 02$ allele had a stronger effect on age of onset than individual HLA-C SNPs, and this allele was highly significant in each of the three individual cohorts studied $(P<0.001)$. In our study population, psoriasis patients with two copies of $H L A-C * 06: 02$ developed psoriasis nearly 12 years earlier than those without $H L A-C * 06: 02$. This is likely to underestimate the true effect of $H L A-C * 06: 02$ because our study population contained relatively more type I psoriasis (onset < age 40) than type II psoriasis (onset $>$ age 40) (Supplementary Figures 1-4), where it has been shown that $C * 06: 02$ is more common in type I psoriasis compared to type II psoriasis $[1,3,4]$. The SNP rs17716942 in the innate antiviral gene IFIH1 was also significant in the meta-analysis $(P=0.002$, FDR $<$ 0.05). IFIH1 encodes a cytoplasmic helicase that acts as a sensor for double-stranded RNA. However, this SNP was not significantly associated with age of onset when examined in an independent cohort, thus further examination of this gene in additional cohorts is warranted.

Genetic variations in ERAP1, IL23R, and LCE3B/LCE3C have recently been associated with pediatric-onset psoriasis (onset < age 18) in a small study [18]. However, in that study the same variants were also shown to be associated with adult-onset psoriasis, suggesting, they may not be specific to pediatric-onset psoriasis. In our study, $L C E 3 B / L C E 3 C$ was significantly associated with age of onset in the GAIN cohort $(P=0.007)$, but not in the meta-analysis (FDR $=0.17)$. We did not observe any evidence for association of ERAP1 or IL23R in our data.

Considering that $H L A-C * 06: 02$ has a much larger effect size (odds ratio $>3.0$ ) on the risk of developing psoriasis compared to the other psoriasis risk alleles (odds ratios $<2.0$ ), it is possible that some of these other risk alleles have a smaller influence on age of onset that would only be detected in a study of larger sample size. There could also be a cumulative additive effect of psoriasis risk alleles on age of onset. Indeed, it has previously been shown that a genetic risk score that combines the genetic burden of the top ten psoriasis genes is significantly higher in patients who develop psoriasis before age 30 compared to those who develop psoriasis after age 30 [19].

In summary, in a large dataset of 1,498 cases we have examined 39 psoriasis variants for their association with age of psoriasis onset. Only the HLA-C locus demonstrated robust evidence of association with age of onset, with HLA$C * 06: 02$ having the strongest effect. In the meta-analysis a variant in IFIH1 was also found to be significant, however this SNP was not replicated in an independent cohort. Further studies are needed to clarify the role of genetic variants on the age at which psoriasis manifests. 







\section{Authors' Contribution}

Yingchang Lu and Sinae Kane contributed equally to this work.

\section{Acknowledgments}

The GAIN dataset used for the analyses described in this paper was obtained from the database of Genotypes and Phenotypes (dbGaP) found at http://www.ncbi.nlm.nih.gov/gap/ through $\mathrm{dbGaP}$ accession number phs000019.v1.p1. Samples and associated phenotype data for the Collaborative Association Study of Psoriasis were provided by Drs. James T. Elder (University of Michigan, Ann Arbor, MI), Gerald G. Krueger (University of Utah, Salt Lake City, UT), Anne Bowcock (Washington University, St. Louis, MO), and Gonçalo R. Abecasis (University of Michigan, Ann Arbor, MI). This project has been funded in part by federal funds from the National Institutes of Health under Award 5K08AR057763 to Wilson Liao.

\section{References}

[1] T. Henseler and E. Christophers, "Psoriasis of early and late onset: characterization of two types of psoriasis vulgaris," Journal of the American Academy of Dermatology, vol. 13, no. 3, pp. 450-456, 1985.

[2] C. Ferrándiz, R. M. Pujol, V. García-Patos, X. Bordas, and J. A. Smandía, "Psoriasis of early and late onset: a clinical and epidemiologic study from Spain," Journal of the American Academy of Dermatology, vol. 46, no. 6, pp. 867-873, 2002.

[3] M. H. Allen, H. Ameen, C. Veal et al., "The major psoriasis susceptibility locus PSORS1 is not a risk factor for late-onset psoriasis," Journal of Investigative Dermatology, vol. 124, no. 1, pp. 103-106, 2005.

[4] J. E. Guojónsson, A. Kárason, A. A. Antonsdóttir et al., "HLACw6-positive and HLA-Cw6-negative patients with psoriasis vulgaris have distinct clinical features," Journal of Investigative Dermatology, vol. 118, no. 2, pp. 362-365, 2002.

[5] F. Capon, M.-J. Bijlmakers, N. Wolf et al., "Identification of ZNF313/RNF114 as a novel psoriasis susceptibility gene," Human Molecular Genetics, vol. 17, no. 13, pp. 1938-1945, 2008.

[6] Y. Liu, C. Helms, W. Liao et al., "A genome-wide association study of psoriasis and psoriatic arthritis identifies new disease loci," PLoS Genetics, vol. 4, no. 3, Article ID e1000041, 2008.

[7] R. P. Nair, K. C. Duffin, C. Helms et al., "Genome-wide scan reveals association of psoriasis with IL-23 and NF-kappaB pathways," Nature Genetics, vol. 41, no. 2, pp. 199-204, 2009.

[8] P. E. Stuart, R. P. Nair, E. Ellinghaus et al., "Genome-wide association analysis identifies three psoriasis susceptibility loci," Nature Genetics, vol. 42, no. 11, pp. 1000-1004, 2010.

[9] E. ELinghaus, D. ELinghaus, P. E. Stuart et al., "Genome-wide asociation study identifies a psoriasis susceptibility locus at TRAF3IP2," Nature Genetics, vol. 42, no. 11, pp. 991-995, 2010.

[10] U. Hüffmeier, S. Uebe, A. B. Ekici et al., "Common variants at TRAF3IP2 are associated with susceptibility to psoriatic arthritis and psoriasis," Nature Genetics, vol. 42, no. 11, pp. 996-999, 2010.

[11] A. Strange, F. Capon, C. C. Spencer et al., "A genome-wide association study identifies new psoriasis susceptibility loci and an interaction between HLA-C and ERAP1," Nature Genetics, vol. 42, no. 11, pp. 985-990, 2010.

[12] R. de Cid, E. Riveira-Munoz, P. L. J. M. Zeeuwen et al., "Deletion of the late cornified envelope LCE3B and LCE3C genes as a susceptibility factor for psoriasis," Nature Genetics, vol. 41, no. 2, pp. 211-215, 2009.

[13] L. C. Tsoi, S. L. Spain, J. Knight et al., "Identification of 15 new psoriasis susceptibility loci highlights the role of innate immunity," Nature Genetics, vol. 44, no. 12, pp. 1341-1348, 2012.

[14] J. T. Elder, A. T. Bruce, J. E. Gudjonsson et al., "Molecular dissection of psoriasis: integrating genetics and biology," Journal of Investigative Dermatology, vol. 130, no. 5, pp. 1213-1226, 2010.

[15] B. N. Howie, P. Donnelly, and J. Marchini, "A flexible and accurate genotype imputation method for the next generation of genome-wide association studies," PLoS Genetics, vol. 5, no. 6, Article ID e1000529, 2009.

[16] P. I. de Bakker, M. A. R. Ferreira, X. Jia, B. M. Neale, S. Raychaudhuri, and B. F. Voight, "Practical aspects of imputationdriven meta-analysis of genome-wide association studies," Human Molecular Genetics, vol. 17, no. R2, pp. R122-R128, 2008.

[17] H. Chen, G. Hayashi, O. Y. Lai et al., "Psoriasis patients are enriched for genetic variants that protect against HIV-1 disease," PLoS Genetics, vol. 8, no. 2, Article ID e1002514, 2012.

[18] J. G. Bergboer, A. M. Oostveen, M. E. de Jager et al., "Paediatriconset psoriasis is associated with ERAP1 and IL23R loci, LCE3C_LCE3B deletion and HLA-C*06," British Journal of Dermatology, vol. 167, no. 4, pp. 922-925, 2012.

[19] H. Chen, A. Poon, C. Yeung et al., "A genetic risk score combining ten psoriasis risk loci improves disease prediction," PLoS ONE, vol. 6, no. 4, Article ID e19454, 2011. 


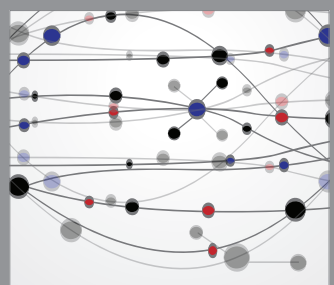

The Scientific World Journal
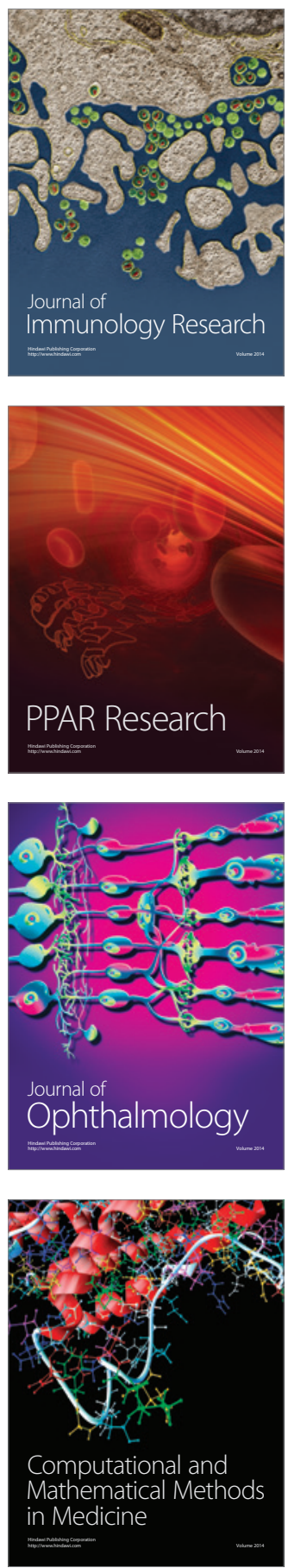

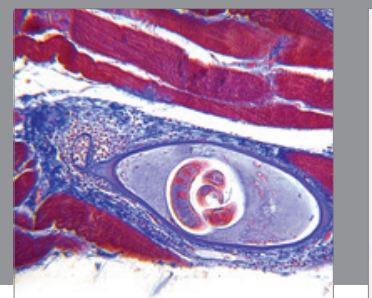

Gastroenterology

Research and Practice
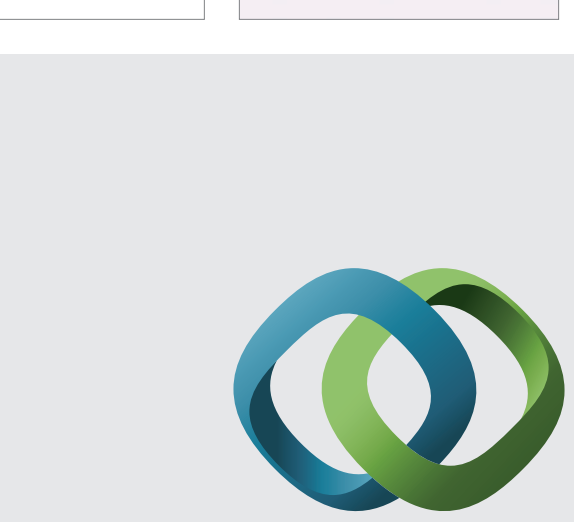

\section{Hindawi}

Submit your manuscripts at

http://www.hindawi.com
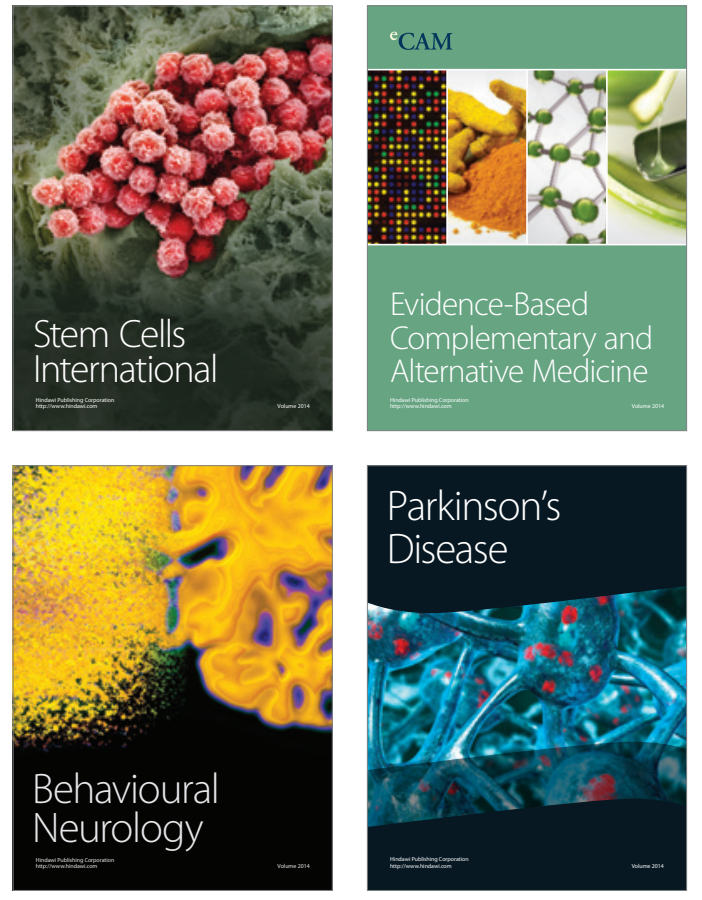
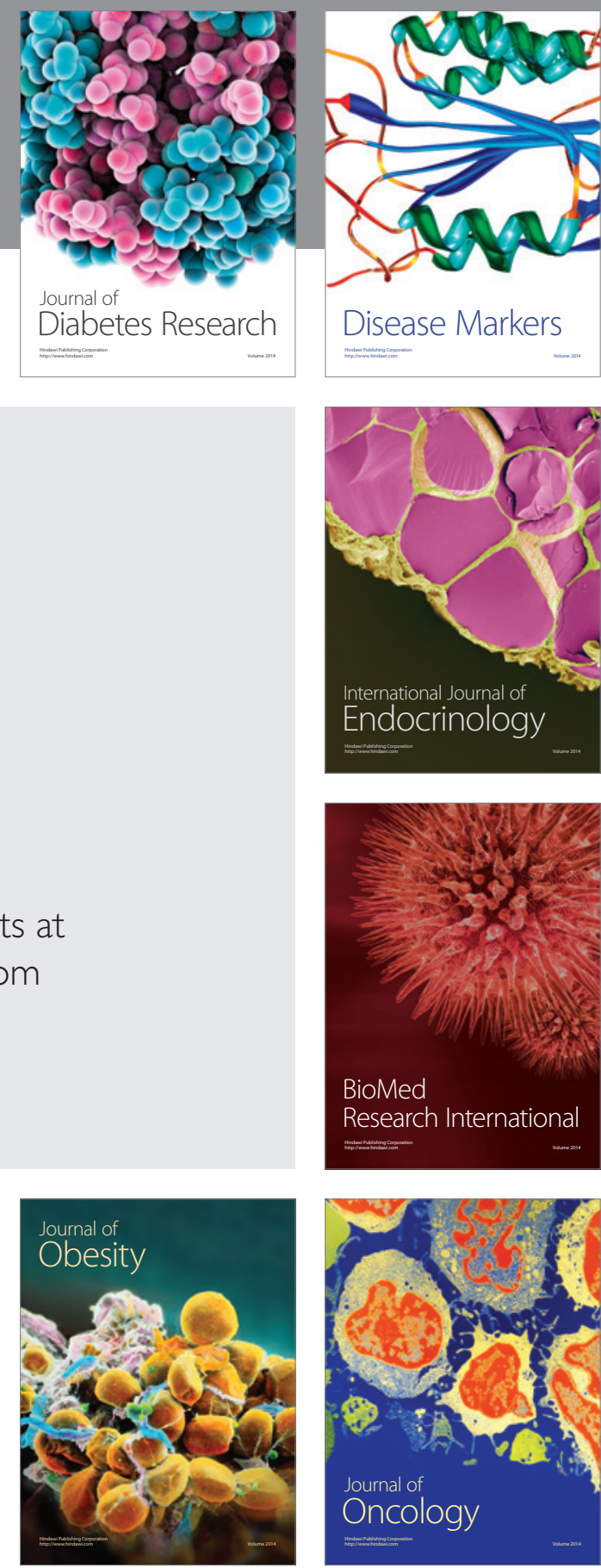

Disease Markers
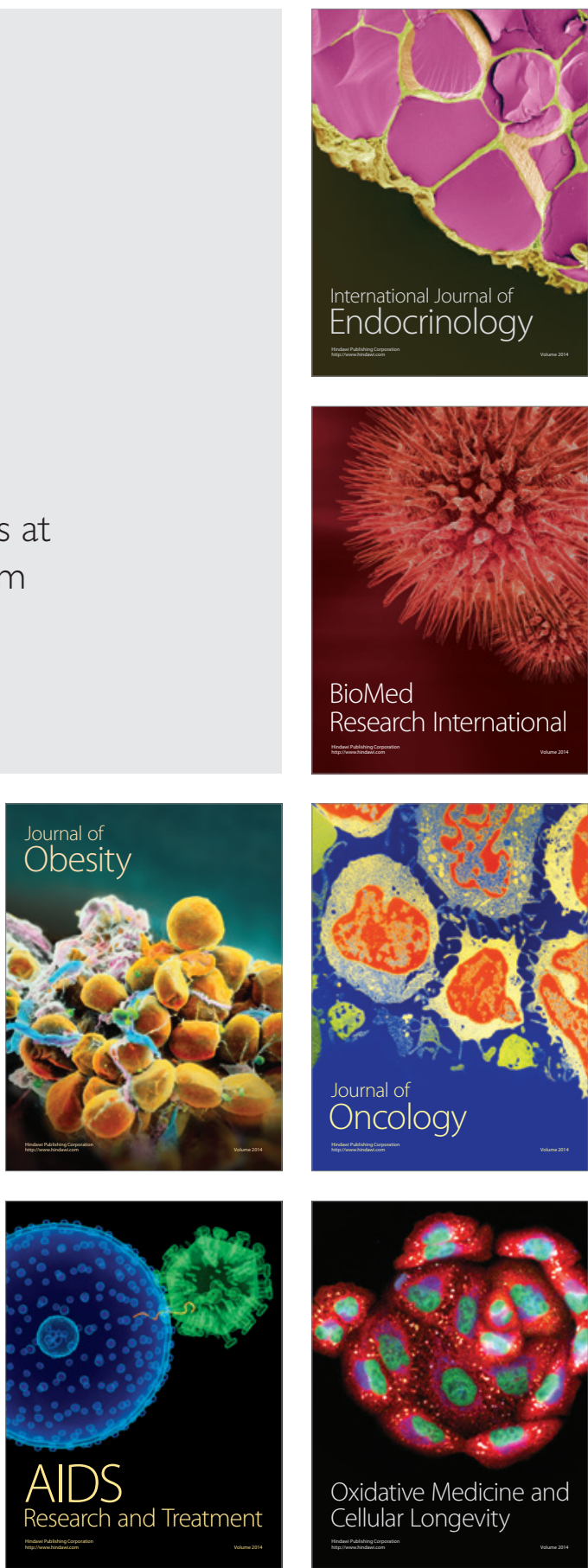